\title{
Giant magnetocaloric effect in composites based on polymeric matrix and manganese arsenide
}

\author{
Nikolay Pankratov ${ }^{1, \star}$, Victor Mitsiuk², Alexander Vetcher ${ }^{2}$, Andrei Krokhotin $^{1}$, Gennadiy Govor $^{2}$, and Sergey Nikitin ${ }^{1}$ \\ ${ }^{1}$ Lomonosov Moscow State University, Physics faculty, Leninskie Gory, 119991 Moscow, Russia \\ ${ }^{2}$ Scientific-Practical Material Research Centre of the National Academy of Science of Belarus, P. Brovki 19, 220072 Minsk, Belarus
}

\begin{abstract}
A field and/or temperature hysteresis is more than just an interesting incomprehensibility that occurs in materials with a first-order magnetic transition. Indeed, the reversibility of the magnetocaloric effect (MCE), being essential for magnetic heat pumps, strongly depends on the width of the thermal hysteresis and, therefore, it is necessary to find solutions to minimize losses associated with thermal hysteresis in order to maximize the efficiency of magnetic cooling devices. In this work, the polymer matrix composites with MnAs powder as reinforcing material were obtained and its MCE by direct method was studied. The influence of composite preparation process on the MCE was investigated. It was found that the MCE of bulk MnAs shows strong temperature hysteresis caused by magnetostructural transition from paramagnetic to ferromagnetic state, which also leads to an irreversible MCE at cooling at first magnetization. The reversible MCE of bulk MnAs in field $12.5 \mathrm{kOe}$ is $\Delta T \sim 0.4 \mathrm{~K}$. It was shown that Mn-based polymeric composite cured in aligned magnetic field presents a reinforcement of the MCE temperature hysteresis. Thus, the MCE value in aligned composite is less than the value of the MCE in the bulk MnAs. It was shown that the decreasing of linear size of MnAs grains in the polymeric composite leads to sharp decreasing of the MCE to value of $0.05 \mathrm{~K}$. It was established that the optimal properties, such as giant MCE and low temperature hysteresis, are found for composite hardened under pressure of $10 \mathrm{kBar}$. In this composite an increase of reversible MCE (compared to the bulk MnAs) till to the value of $\Delta T \sim 1.2 \mathrm{~K}$ was observed.
\end{abstract}

\section{Introduction}

The composite material which is made of metallic and polymeric material by certain processes, can retain the advantages of the original components, overcome some shortcomings and show some new properties. A composite material is a multi-phase system consisted of matrix material and reinforcing material. Matrix material is a continuous phase, and reinforcing material is a dispersed phase, such as fiber, sheet or particle. The composite not only retains the key feature of the original component materials, but also gets the performances that are not depicted by the original components through the combined effects [1].

Various functional materials can be produced by compounding different materials of function. At present, electromagnetic functional materials develop most rapidly and are used most commonly as the functional composite materials. These composite materials can be processed into magnetic products with the required shape and the certain mechanical properties by the general forming methods (such as compression molding, laminating, etc.) of polymeric materials. In order to obtain a good overall performance, we must increase the amount of filler or enhanced material as much as possible, control the interface of composite materials and make composite materials have better process ability [2].

\footnotetext{
^e-mail: pankratov@phys.msu.ru
}

Magnetic refrigeration technology is a rapidly developing technology that is assumed to be capable of competing with and hopefully surpassing traditional vapourcompression refrigeration in terms of efficiency, device volume and ecological impact in the near future [3]. From this point of view, magnetic compounds exhibiting large magnetocaloric effects (MCE) in the temperature range 270-320 K (under magnetic field of 10-20 kOe) are attracting much attention due to their potential application in room temperature magnetic refrigeration [4-8]. The search for new magnetocaloric materials as well as the optimization of known materials is a growing field of interest. The new magnetic materials showing a large MCE with narrow hysteresis, particularly around room temperature, are the subject of intensive research activity.

During the past years, materials undergoing the firstorder magnetic transition (FOMT) have attracted considerable attention. In these compounds, many fascinating functional properties, such as MCE [9], magnetic-fieldinduced shape recovery [10] and magnetoresistance (MR) [11], were gained near FOMT. It is know, FOMTs can be divided into two categories [12]. One is magnetoelastic transition, in which no crystal symmetry evolution but the change of lattice parameters is observed on both sides of the phase transition, such as $\mathrm{MnFeP}_{1-x} \mathrm{As}_{x}$ and $\mathrm{La}(\mathrm{Fe}, \mathrm{Si})_{13}$ [13-15]. The other is magnetostructural transition (MST), in which magnetic and crystallographic transitions oc- 
cur simultaneously, such as $\mathrm{Gd}_{5}\left(\mathrm{Si}_{2} \mathrm{Ge}_{2}\right), \mathrm{Mn}-\mathrm{Ni}-\mathrm{Ga}(\mathrm{Fe})$ $[16,17]$ and MnAs [18-20]. In these cases, relatively smaller thermal/magnetic hysteresis than those possessing magnetostructural transition are generally revealed. In numerous materials presenting FOMT, Mn-based intermetallic compounds, such as $\mathrm{MnFePAs}(\mathrm{Si} / \mathrm{Ge})[13,18]$, MnAs [20, 21], Ni-Mn-based [9-11], and $\mathrm{Mn}(\mathrm{Co}, \mathrm{Ni}) \mathrm{Ge}$ systems $[12,14]$, have extensively been investigated owing to the low cost and higher magnetic moment of Mn atoms, sometimes even $4 \mu_{\mathrm{B}}$ per Mn atom [18].

The large MCE and giant MR have to observe for Mnbased alloys, because such alloys often demonstrate the transitions with abrupt change of magnetisation or conductivity. It take notice, that magnetic properties in Mn-based alloys connect with interatomic Mn-Mn distance, thereby the contemporary investigation give its up to regulate the Mn-Mn interatomic distance and attain the steep change of magnetisation respectively, as for instance at partial atom replacement $[12,17]$ or by external pressure [22]. So, the alloys and composites based on MnAs have attracted attention during several decade.

The MnAs compound is a ferromagnetic with magnetic moment equal to $3.4 \mu_{\mathrm{B}}$ per $\mathrm{Mn}$ atom [23]. It displays one of the highest MCE neighboring room temperature. At the Curie temperature $\left(T_{\mathrm{C}}\right) \mathrm{MnAs}$ demonstrates the MST from low-temperature ferromagnetic hexagonal NiAs-type state to a high-temperature paramagnetic state with orthorhombic MnP-type crystal structure [19, 24]. These MST and their concomitant entropy changes can be triggered by applying an external magnetic field $[19,24]$ and by an external pressure [25] giving rise to the MCE. It was shown that both ferro- and paramagnetic metastable phase coexist in MnAs in the region of phase transition, and so the MCE is caused by field induced magnetic phase transition from paramagnetic to ferromagnetic state [19].

The aim of this paper is to investigate the influence of both the polymeric matrix type and the preparation of the composites on the MCE temperature behaviour and finding the MCE dependence on unstable magnetic condition produced by thermal and magnetic history of sample. The submicron particles composite was also investigated. The MCE study was performed by direct measurements of temperature shift at an adiabatic change of magnetic field.

\section{Sample preparation and experimental technique}

Single crystals of equiatomic manganese arsenide were grown using the Bridgman-Stockbarger technique from a melt of the corresponding composition in evacuated quartz ampoules with a conical tip. The ampoule was dropped from the melting zone along the crystallization zone at a rate of $2-3 \mathrm{~mm} / \mathrm{h}$.

The crystallographic quality of the alloys was determined using a powder X-ray diffraction method (XRD) using $\mathrm{Cu} \mathrm{K}_{\alpha}$ radiation. The indexing procedure was applied to the obtained diffraction patterns in order to extract relevant crystallographic parameters. The X-ray emission analysis of the grown single crystals demonstrates that the

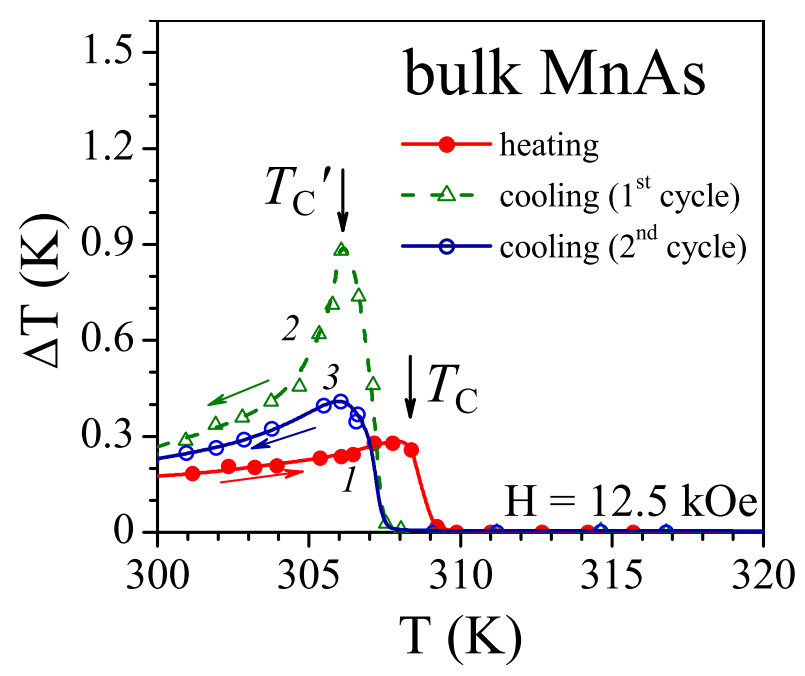

Figure 1. The MCE temperature dependencies of MnAs compound in field $12.5 \mathrm{kOe}$ at heating (1) and cooling (2 and 3). Curve 2 is the irreversible MCE at first magnetization.

deviation from the stoichiometric composition did not exceed $\Delta x<0.01$ at $\%$. The main phase was identified to be a hexagonal NiAs-type crystal structure with $\mathrm{P} 6 / \mathrm{mmm}$ symmetry. The lattice parameters were closes to the ones reported for pure MnAs, e.g. $a=3.722 \AA$ and $c=5.699 \AA$. The microstructure was examined using metallography.

The composites were prepared from microsrystalline and submicrosrystalline metallic powder fixed in polymeric matrix; the volume of polymer does not exceed $8 \%$. The grain sizes was examined by electronic microscope. There were obtained both aligned by magnetic field and isotropic composites hardened under hydrostatic pressure up to $20 \mathrm{kbar}$.

The MCE was measured by direct method by detecting the temperature as function of the magnetic field change. The temperature was detected by the copper-constantan thermocouple. The MCE was studied in the temperature range of $100-350 \mathrm{~K}$ in magnetic field up to $H=12.5 \mathrm{kOe}$.

\section{Results and discussion}

The influence of composite preparation process on the MCE was studied. In this frame we choice three ways to manage the MCE behaviour of composites. One is to study the MCE dependence on the magnetic texture of composite. In this case the viscous mixture of MnAs powder and liquid polymer was hardened in uniform magnetic field produced by two permanent magnets. Second an influence of the magnetoactive material grain sizes on the MCE of composite was studied. The composite with micro- and submicrosizes grains were obtained and its MCE was studied. Other is the effect of the hydrostatic pressure applied at the composite's moulding process.

Fig. 1 shows the MCE temperature dependencies in bulk MnAs obtained at adiabatic field excursion from zero to the value of $12.5 \mathrm{kOe}$ in the region of magnetic phase transition. Curve 1 relates to the case where the sample was heated up from ferromagnetic state $(T<300 \mathrm{~K})$ 


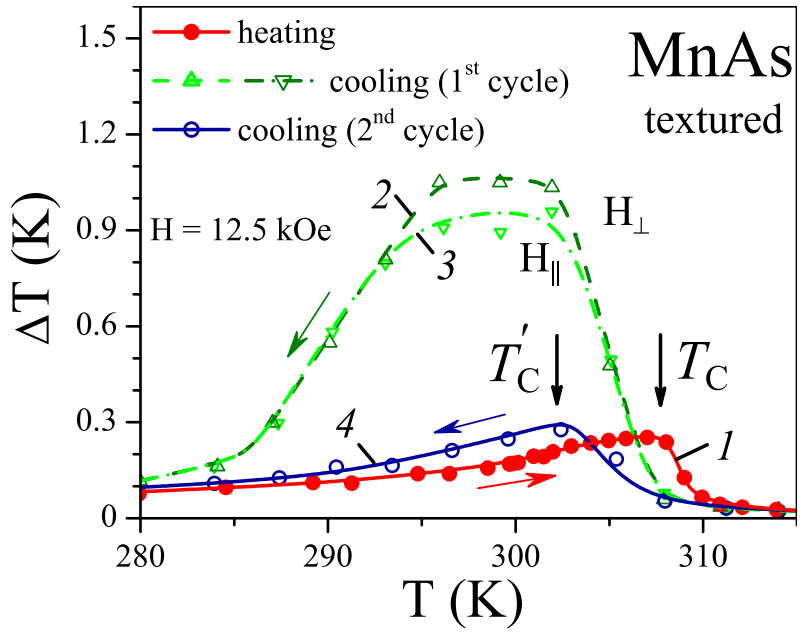

Figure 2. The MCE temperature dependencies of field aligned MnAs-based composite in magnetic field of $12.5 \mathrm{kOe}$ at heating (1) and cooling (2,3, and 4). Curves 2 and 3 demonstrate the irreversible MCE at first magnetization for two different sample orientation in the external magnetic field.

and above the temperature of phase transition the sample reaches the paramagnetic state. It was found that in bulk MnAs on the heating the MCE reaches maximum value of $\Delta T=0.28 \mathrm{~K}$ (in field of $12.5 \mathrm{kOe}$ ) at temperature $T=308 \mathrm{~K}$

In second case (curve 2) the sample is cooled from paramagnetic state $(T>320 \mathrm{~K})$ to room temperature, the MCE shows maximum value of $\Delta T=0.88 \mathrm{~K}$ at temperature $T=306 \mathrm{~K}$. Our data point to the fact that there are two metastable phase caused by transition from ferromagnetic to paramagnetic state [19]. As far as relative ratio of ferromagnetic and paramagnetic phases in the samples at heating and cooling is distinct, the MCE has different values depending on thermal history of the sample. This conclusion also is supported by the curve 3 on fig. 1 displayed the MCE behaviour of the preliminary magnetised sample. A first magnetic field switch on causes large MCE (curve 2) and changes relation between ferromagnetic and paramagnetic phases in comparison with the initial state. Obviously at the second magnetization the magnitude of MCE is less than at first one, because the MCE is caused by the transition from paramagnetic to ferromagnetic state and the amount of paramagnetic phase decreases after the first switching field.

Fig. 2 shows the MCE temperature dependencies in magnetic field change of $12.5 \mathrm{kOe}$ in textured composite. This composite properties are texture-specific because there is an axis of preferred orientation of spontaneous magnetization of the grains. By this reason the MCE temperature dependencies at cooling and at heating were studied under the two orientation of magnetic field, along and perpendicular to texture axis, respectively. The maxima value of $\mathrm{MCE}$ at the heating is $\Delta T=0.3 \mathrm{~K}$ at temperature of $T_{\mathrm{C}}=309 \mathrm{~K}$. There is no any anisotropy of the MCE at heating of the field oriented composite.

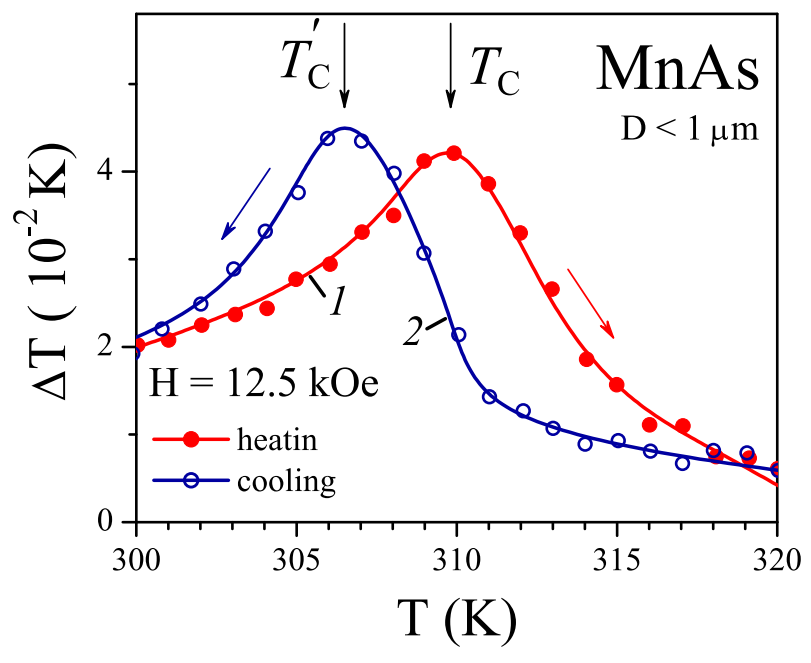

Figure 3. The MCE temperature dependencies of submicron's MnAs-based composite in field of $12.5 \mathrm{kOe}$ at heating (1) and cooling (2).

It is found that at cooling the MCE temperature dependence demonstrates a broad maximum in temperature range $296-302 \mathrm{~K}$ with maximum value of $\Delta T=1 \mathrm{~K}$, however it is irreversible MCE caused by magnetostructural transition from paramagnetic to ferromagnetic state. The MCE of the preliminary magnetized sample is quit less and comparable with MCE at heating (see curves 1 and 4). The temperature of maximum of MCE at cooling is found of $T_{C}^{\prime}=302 \mathrm{~K}$. It is noticed, that at cooling there is an influence of magnetic anisotropy of the MCE. In case of the field applied perpendicular to textured axis the MCE value is a bit large than in case where the direction of magnetic field coincide with textured axis. Here, $\Delta T_{\perp}$ is equal to $1.05 \mathrm{~K}$ and $\Delta T_{\|}=0.95 \mathrm{~K}$. At the magnetization of preliminary magnetized sample the anisotropy of MCE is disappear. It can be conclude that the composite aligned by magnetic field demonstrates an increasing a MCE temperature hysteresis compared with bulk MnAs, while the value of reversible MCE at heating and cooling go up in this composite.

Fig. 3 shows the MCE temperature dependencies of the MnAs-based composite with submicrosizes powder in cases of heating and cooling. On both dependencies the MCE value does not exceed of $\Delta T=0.05 \mathrm{~K}$. So, it is seen that the decreasing of the magnetoactive material grain size in the MnAs-based composite to the value less than $1 \mu \mathrm{m}$ leads to strong decreasing of the MCE compared with bulk MnAs. The temperatures of the MCE maximum $T_{\mathrm{C}}^{\prime}=306 \mathrm{~K}$ and $T_{\mathrm{C}}=309 \mathrm{~K}$ on the cooling and the heating, respectively. So, the temperature hysteresis of MCE preserves in submicron composite.

Fig. 4 displays temperature dependence of the MCE of the composite obtained under hydrostatic pressure of $10 \mathrm{kbar}$. It is seen that this composite shows a quasireversible MCE behaviour. The maximum of MCE $(\Delta T=1.3 \mathrm{~K})$ is reached at $T_{\mathrm{C}}=309 \mathrm{~K}$ for both cooling and heating. The MCE value in this composite is exceed the MCE in bulk MnAs at the same magnetic field [19]. 


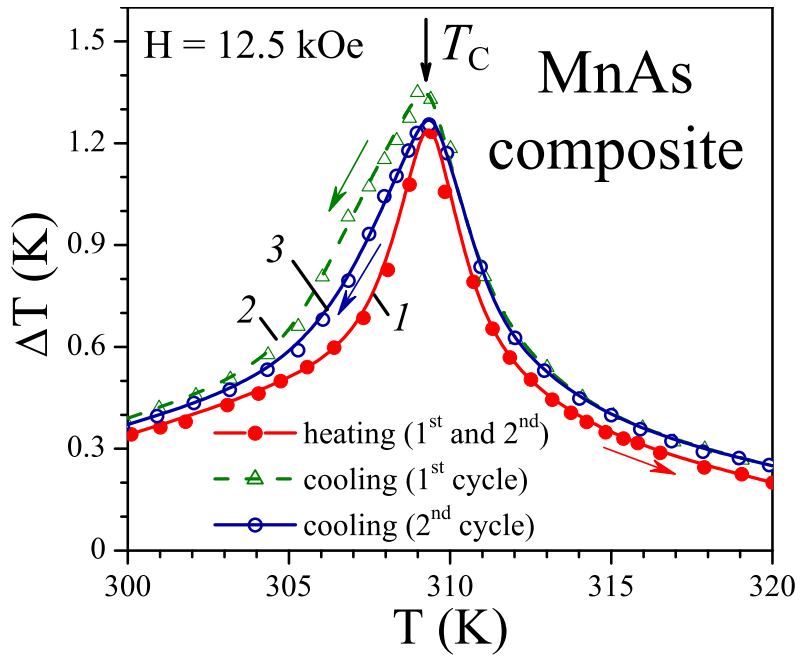

Figure 4. The MCE temperature dependencies of MnAs-based polymer composite cured on pressure $10 \mathrm{kbar}$ at heating (1) and cooling ( 2 and 3 ) in field $12.5 \mathrm{kOe}$. Curve 2 is the irreversible MCE at first magnetization.

It is quite possible that this behaviour is result of residual pressure of polymeric matrix pushed to MnAs grains [25].

\section{Summary}

It was shown, that the decreasing of grain size under $1 \mu \mathrm{k}$ reduces the MCE in submicron's MnAs-based composites. It was found, that both the MCE hysteresis and the MCE maximum value are increased in textured composites compared with bulk MnAs.

It was established that the optimal properties, such as giant MCE and low temperature hysteresis, are found in composite with particle's size of $\sim 1 \mu \mathrm{m}$ and polyvinyl acetate matrix hardened at pressure of $10 \mathrm{kbar}$. The maximum of MCE in this composite is $\Delta T \sim 1.3 \mathrm{~K}$ (at $309 \mathrm{~K}$ ) in field of $12.5 \mathrm{kOe}$. At the same time the temperature hysteresis of MCE is foes down in this composite. These properties are caused by the residual pressure of the polymer mould on the grains.

The work is supported by RFBR grants \# 16-52-00223, \# 160200472 and BRFBR grant \#T16P-170.

\section{References}

[1] F.C. Campbell, Manufacturing Processes for Advanced Composites (Elsevier Science, Amsterdam, 2004), ISBN 978-1-85617-415-2

[2] R.M. Wang, S.R. Zheng, Y.P. Zheng, Polymer Matrix Composites and Technology, Woodhead Publishing Series in Composites Science and Engineering (Woodhead Publishing, 2011), ISBN 978-0-85709221-2

[3] C. Zimm, A. Jastrab, A. Sternberg, V. Pecharsky, K. Gschneidner, M. Osborne, I. Anderson, Description and performance of a near-room temperature magnetic refrigerator (Boston, MA: Springer US, 1998), pp. 1759-1766
[4] A.M. Tishin, Y.I. Spichkin, The magnetocaloric effect and its applications, Series in condensed matter physics (Institute of Physics Pub., Bristol and Philadelphia, 2003), ISBN 0-750-30922-9

[5] O. Gutfleisch, M.A. Willard, E. Brück, C.H. Chen, S.G. Sankar, J.P. Liu, Adv. Mater. 23, 821 (2011)

[6] E. Brück, Magnetocaloric refrigeration at ambient temperature (Elsevier, Amsterdam, The Netherlands, 2007), chap. 4, pp. 235-291

[7] K.A. Gschneidner, V.K. Pecharsky, Int. J. Refrig. 31, 945 (2008)

[8] K.A. Gschneidner, V.K. Pecharsky, Annu. Rev. Mater. Sci. 30, 387 (2000)

[9] J. Liu, T. Gottschall, K.P. Skokov, J.D. Moore, O. Gutfleisch, Nat. Mater. 11, 620 (2012)

[10] R. Kainuma, Y. Imano, W. Ito, Y. Sutou, H. Morito, S. Okamoto, O. Kitakami, K. Oikawa, A. Fujita, T. Kanomota et al., Nature (London) 439, 957 (2006)

[11] H.C. Xuan, L.J. Shen, T. Tang, Q.Q. Cao, D.H. Wang, Y.W. Du, Appl. Phys. Lett. 100, 172410 (2012)

[12] N.T. Trung, V. Biharie, L. Zhang, L. Caron, K.H.J. Buschow, E. Brück, Appl. Phys. Lett. 96, 162507 (2010)

[13] O. Tegus, E. Brück, K.H.J. Buschow, F.R. de Boer, Nature (London) 415, 150 (2002)

[14] F.X. Hu, B.G. Shen, J.R. Sun, Z.H. Cheng, G.H. Rao, X.X. Zhang, Appl. Phys. Lett. 78, 3675 (2001)

[15] I.A. Radulov, K.P. Skokov, D.Y. Karpenkov, T. Gottschall, O. Gutfleisch, J. Magn. Magn. Mater. 396, 228 (2015)

[16] V.K. Pecharsky, J. K. A. Gschneidner, Phys. Rev. Lett. 78, 4494 (1997)

[17] E.K. Liu, W.H. Wang, L. Feng, W. Zhu, G.J. Li, J.L. Chen, H.W. Zhang, G.H. Wu, C.B. Jiang, H.B. Xu et al., Nat. Commun. 3, 873 (2012)

[18] E. Brück, O. Tegus, D.T.C. Thanh, N.T. Trung, K.H.J. Buschow, Int. J. Refrig. 31, 763 (2008)

[19] V.I. Mitsiuk, N.Y. Pankratov, G.A. Govor, S.A. Nikitin, A.I. Smarzhevskaya, Physics of the Solid State 54, 1988 (2012)

[20] N.Y. Pankratov, V.I. Mitsiuk, A.I. Krokhotin, A.I. Smarzhevskaya, G.A. Govor, S.A. Nikitin, V.M. Ryzhkovskii, Solid State Phenomena 190, 343 (2012)

[21] P.F. Xu, S.H. Nie, K.K. Meng, S.L. Wang, L. Chen, J.H. Zhao, Appl. Phys. Lett. 97, 042502 (2010)

[22] S. Niziol, R. Zach, J.P. Senateur, J. Beille, J. Magn. Magn. Mater. 79, 333 (1989)

[23] H. Wada, Y. Tanabe, Appl. Phys. Lett. 79, 3302 (2001)

[24] G.A. Govor, in Double Exchange in Heusler Alloys and Related Materials, edited by K. Baerner (2007), chap. 2, pp. 29-62

[25] S. Gama, A.A. Coelho, A. de Campos, A. Magnus, G. Carvalho, F.C.G. Gandra, P.J. Ranke, N.A. Oliveira, Phys. Rev. Lett. 93, 237202(4) (2004) 